\title{
Robust Fractional-Order Proportional-Integral Observer for Synchronization of Chaotic Fractional-Order Systems
}

\author{
Ibrahima N'Doye, Member, IEEE, Khaled Nabil Salama, Senior Member, IEEE, and \\ Taous-Meriem Laleg-Kirati, Senior Member, IEEE
}

\begin{abstract}
In this paper, we propose a robust fractional-order proportional-integral (FOPI) observer for the synchronization of nonlinear fractional-order chaotic systems. The convergence of the observer is proved, and sufficient conditions are derived in terms of linear matrix inequalities (LMIs) approach by using an indirect Lyapunov method. The proposed (FOPI) observer is robust against Lipschitz additive nonlinear uncertainty. It is also compared to the fractional-order proportional (FOP) observer and its performance is illustrated through simulations done on the fractional-order chaotic Lorenz system.
\end{abstract}

Index Terms-Chaos synchronization, robust proportionalintegral observer design, fractional-order chaotic systems, indirect Lyapunov approach, Linear Matrix Inequality (LMI).

\section{INTRODUCTION}

$\mathbf{F}$ RACTIONAL calculus has gained significant attention in various application fields in engineering and science. Thanks to their non-local properties, fractional-order derivatives provide a more appropriate tool for modeling complex phenomena.

The study of the synchronization problem for nonlinear systems has been very important from nonlinear sciences point of view, in particular the applications to secure data transmission, biology, cryptography and so on [1]. Synchronization of chaos occurs in a process wherein two chaotic systems either equivalent or non equivalent have a common behavior due to coupling or forcing of some properties of their motion [1]. The chaos synchronization problem can be regarded as an observer design procedure, where the coupling signal is viewed as output and the response system as an observer [2]-[5]. Fractional differentiation order has a direct effect on the chaotic behavior of nonlinear dynamical systems and on

This article has been accepted for publication in a future issue of this journal, but has not been fully edited. Content may change prior to final publication.

This work was supported by King Abdullah University of Science and Technology (KAUST), KSA. Recommended by Associate Editor Xiaoming Hu. (Corresponding author: Ibrahima N'Doye.)

Citation: I. N'Doye, K. N. Salama, and T.-M. Laleg-Kirati, "Robust fractional-order proportional-integral observer for synchronization of chaotic fractional-order systems," IEEE/CAA J. of Autom. Sinica, pp. 1-10, 2018. DOI: $10.1109 /$ JAS.2017.7510874.

The authors are all with Computer, Electrical and Mathematical Sciences and Engineering Division (CEMSE), King Abdullah University of Science and Technology (KAUST), Thuwal 23955-6900, Saudi Arabia (e-mail: ibrahima.ndoye@kaust.edu.sa; khaled.salama@kaust.edu.sa; taousmeriem.laleg@kaust.edu.sa).

Color versions of one or more of the figures in this paper are available online at http://ieeexplore.ieee.org. the synchronization process. Indeed the synchronization error decreases as the differentiation order increases [6]. In the same context, a recent study has shown that the control and synchronization are easier with low order fractional systems [1], [7]. A similar result is reported in [8], which indicates that smaller the fractional-order is, better is the synchronization. Different synchronization methods exist for both integer-order systems and fractional order systems (see for example [9] and [10]). Among these methods proposed for integer-order systems are the observers, used usually for state estimation and which have been proposed as an efficient tool for synchronization for example in [3], [11], [12]. Various control schemes for fractional-order chaotic systems have been intensively studied, unfortunately research on the control and synchronization of fractional-order chaotic systems using proportional-integral (PI) observer have not been yet investigated. A PI observer is an observer with an integrating effect which takes care of the asymptotic time behavior [13]. PI observers have several attractive features compared to proportional action and combined with fractional-order they allow more flexibilities via selection of the order. In general, a PI observer allows good time recovery at low frequencies whereas the usual proportional $(\mathrm{P})$ observer allows good recovery only in the limit at low frequencies [13].

The main advantage of describing system in fractional-order than normal one in term of observer design is its additional degree of freedom that helps in improving the synchronization errors performance. It is interesting to find that the fractionalorder derivatives and the PI observer gains can be well chosen appropriately to adjust the synchronization effect. The proposed fractional-order PI observer can be applied to a wide class of chaotic fractional systems and it may also enhance security in communication and chaotic encryption schemes due to its complexity.

We propose to extend the idea of observer-based synchronization to fractional chaotic systems. In this context, we propose a robust fractional-order proportional integral (FOPI) observer for a class of nonlinear fractional-order chaotic systems which provides very interesting flexibility and robustness properties with respect to perturbations. The proposed observer uses both proportional and integral corrections allowing for better robustness properties. The design of the observer and its proof of convergence rely on a frequency distributed fractional integrator equivalent model which allows the use of Lyapunov method [14]. This approach has been successfully used in 
several cases including the adaptive parameter estimation problems for some classes of linear fractional-order processes [15] and the observer design for fractional-order nonlinear systems [16]-[18]. However, few studies have addressed robust observer design for fractional-order systems. The convergence of the proposed observer is studied using linear matrix inequalities based on Lyapunov approach. We show that the FOPI observer allows the attenuation of modeling uncertainties and noisy measurements. A comparison of the proposed FOPI observer to the fractional-order proportional (FOP) observer is also studied via simulation examples.

This paper is organized as follows. In section II, some preliminary results are presented. In section III, the problem is formulated. In section IV, the main results are derived, and then sufficient conditions for the asymptotic stability of the fractional-order observer error dynamics are derived in terms of linear matrix inequalities (LMIs). The robustness analysis against nonlinear uncertainty in which an explicit tolerable bound is derived, is performed in section V. In section VI, an example is presented to illustrate the proposed results. Finally, some conclusions are drawn in section VII.

Notations. $M^{T}$ is the transpose of $M$ and $D^{\alpha}$ represents initialized $\alpha^{\text {th }}$ order differintegration. In symmetric block matrices, the operator - represents a term induced by symmetry.

\section{PRELIMINARIES}

In this section, we present some basic definitions and properties of the fractional integration and differentiation. Fractional-order integration and differentiation are the generalization of their integer-order counterparts. Traditional integerorder definitions have been extended to the fractional cases leading to different definitions [19], [20]. One of the primary functions of fractional calculus is Euler's Gamma function defined by the integral

$$
\Gamma(z)=\int_{0}^{\infty} e^{-t} t^{z-1} \mathrm{~d} t
$$

which converges in the right half of the complex plane, i.e $\operatorname{Re}(z)>0$. The definition of fractional integral of continuous function $f(t)$ with respect to $t$ and the terminal value $t_{0}$ is given by [19]

${ }_{t_{0}} D_{t}^{-\alpha} f(t)=\frac{1}{\Gamma(\alpha)} \int_{t_{0}}^{t}(t-\tau)^{\alpha-1} f(\tau) \mathrm{d} \tau, \quad \alpha>0$,

where $\Gamma($.$) is the well-known Gamma function which is$ defined in (1).

The Riemann-Liouville derivative is defined by [19]

$$
{ }_{t_{0}}^{R L} D_{t}^{\alpha} f(t)=D_{t_{0}}^{n} D_{t}^{\alpha-n} f(t), \quad n-1<\alpha<n,
$$

where $n \in \mathbb{Z}^{+}$and $D^{n}$ is the classical $n$-th order derivative.

A fractional-order system can be represented by an infinitedimensional system generally called "diffusive representations" [21]-[24]. In the following we recall briefly a continuous frequency distributed representation of a fractionalorder system. Other equivalent representations can be used as described in [23].
Let $h(t)$ be the impulse response of a linear system. The diffusive representation (or frequency weighting function) of $h(t)$ is called $\mu(\omega)$ with the following relation [14]

$$
h(t)=\int_{0}^{\infty} \mu(\omega) e^{\omega t} \mathrm{~d} \omega,
$$

where $\omega$ is the elementary frequency.

The fractional order integral operator $t_{0} D_{t}^{-\alpha} f(t)$ can be written as [14]

$$
t_{0} D_{t}^{-\alpha} f(t)=h(t) * f(t)
$$

where $*$ denotes the convolution operator and $h(t)=\frac{t^{\alpha-1}}{\Gamma(\alpha)}$, while the diffusive representation of $h(t)$ is defined as

$$
\mu(\omega)=\frac{\sin (\alpha \pi)}{\pi} \omega^{-\alpha} .
$$

In the rest of this paper, $D^{\alpha}$ is used to denote the RiemannLiouville fractional derivative of order $\alpha$.

Lemma 1: The fractional-order nonlinear differential equation [14], [23]

$$
{ }_{t_{0}} D_{t}^{\alpha} x(t)=f(x(t)),
$$

due to the continuous frequency distributed model of the fractional integrator, can be written as

$$
\left\{\begin{array}{l}
\frac{\partial z(\omega, t)}{\partial t}=-\omega z(\omega, t)+f(x(t)) \\
x(t)=\int_{0}^{\infty} \mu(\omega) z(\omega, t) \mathrm{d} \omega
\end{array}\right.
$$

where $z(\omega, t)$ is the infinite dimensional distributed state variable and $\mu(\omega)$ is defined in (6).

An approximation has been proposed to avoid the infinite dimensional nature of the diffusive representation where the frequency domain is truncated to be limited [23]. In the following, we recall this approximation briefly, more details on the convergence and the procedure of the approximation can be found in [23]. Consider a finite network of $N$ frequency points : $\omega=\left[\omega_{1}=\omega_{\min }, \cdots, \omega_{\mathrm{N}}=\omega_{\max }\right]$ with $\omega_{\min }>0$ for $i=1, \cdots, N$ where $N$ is the number of approximation nodes. An approximation of the state $z(\omega, t)$ denoted as $\bar{z}(\omega, t)$ is given via the interpolating functions $\psi_{i}$ as follows:

$$
\bar{z}(\omega, t)=\sum_{i=1}^{N} \psi_{i}(\omega) z\left(\omega_{i}, t\right),
$$

where $z\left(\omega_{i}, t\right)$ is the real state at frequency $\omega_{i}$ and $\psi_{i}(\omega)$ are chosen such that $\bar{z}\left(\omega_{i}, t\right)=z\left(\omega_{i}, t\right)$.

The approximation of the pseudo-state $x(t)$ is given by

$$
\bar{x}(t)=\int_{\omega_{\min }}^{\omega_{\max }} \mu(\omega) \bar{z}(\omega, t) \mathrm{d} \omega .
$$

The accuracy of this approximation depends on the choice of the interpolation functions and the sampling frequencies. An appropriate choice of $\psi_{i}(\omega)$ and for an admissible input $f, \bar{z}(\omega, t)$ and $\bar{x}(t)$ converge to $z(\omega, t)$ and $x(t)$ respectively [23]. 


\section{PRoblem Formulation}

Consider the following nonlinear fractional-order system with external disturbances

$$
\left\{\begin{array}{l}
D^{\alpha} x(t)=A x(t)+\Phi(x(t), u(t))+F d(t), \quad 0<\alpha<1 \\
y(t)=C x(t)
\end{array}\right.
$$

where $x(t) \in \mathbb{R}^{n}$ is the pseudo-state vector, $u(t) \in \mathbb{R}^{m}$ is the control input, $y(t) \in \mathbb{R}^{p}$ is the measurable output and $d(t) \in \mathbb{R}^{q}$ is a bounded unknown input disturbance. $A \in$ $\mathbb{R}^{n \times n}, C \in \mathbb{R}^{m \times n}$ and $F \in \mathbb{R}^{n \times q} . \alpha_{i}=\left[\alpha_{1}, \alpha_{2}, \cdots, \alpha_{n}\right]$ are the fractional orders for $0<\alpha<1$. An interesting situation is when $\alpha_{1}=\alpha_{2}=\cdots=\alpha_{n}$ and in this case system (11) is called commensurate system, otherwise it is non-commensurate system.

For the rest of the paper, we consider the following assumption.

Assumption 1: The nonlinear fractional-order system (11) satisfies the following conditions.

i) The nonlinear vector function $\Phi(x(t), u(t))$ is assumed to be Lipschitz with respect to $x$, uniformly in $u$ and $\Phi\left(0, u^{*}\right)=0$ for all admissible control signal $u^{*}$, i.e

$$
\left\|\Phi\left(x_{1}, u^{*}\right)-\Phi\left(x_{2}, u^{*}\right)\right\| \leqslant \lambda_{1}\left\|x_{1}-x_{2}\right\|
$$

where $\lambda_{1}$ is a Lipschitz constant.

ii) The matrix pair $(C, A)$ is observable and the triplet $(A, F, C)$ is minimum phase [25], [26] i.e. $\forall \sigma \in \mathbb{C}, i=$ $1, \cdots, n$,

$$
\operatorname{rank}\left[\begin{array}{cc}
\sigma I_{n}-A & F \\
C & 0
\end{array}\right]=n+\operatorname{rank}(F),|\arg (\sigma)| \leqslant \alpha_{i} \frac{\pi}{2}
$$

The following lemma proved in [27] will be used in the next section.

Lemma 2: [27] For any matrices $X$ and $Y$ with appropriate dimensions, we have for any $\delta>0$

$$
X^{T} Y+Y^{T} X \leqslant \varepsilon_{1} X^{T} X+\varepsilon_{1}^{-1} Y^{T} Y .
$$

\section{Robust FOPI OBSERVER DESIGN}

In this section, sufficient conditions for the convergence of the fractional-order proportional-integral (FOPI) observer are derived in terms of linear matrix inequalities (LMIs) formulation. The controller gains are determined from feasible solutions of the LMI.

We consider the nonlinear fractional-order proportionalintegral observer in the following form

$$
\left\{\begin{array}{l}
D^{\alpha} \hat{x}(t)=A \hat{x}(t)+\Phi(\hat{x}(t), u(t))+L(y(t)-C \hat{x}(t))+K \xi(t), \\
D^{\alpha} \xi(t)=-T \xi(t)+H(y(t)-C \hat{x}(t)),
\end{array}\right.
$$

where $0<\alpha<1, \hat{x}(t) \in \mathbb{R}^{n}$ is the estimated state vector, $L$ and $K$ are the proportional and the integral observer gains, respectively. The additional state vector $\xi(t) \in \mathbb{R}^{m}$ is the filtered output observer-based synchronization error and matrices $T$ and $H$ are unknown matrices of appropriate dimensions, which must be determined such that $\hat{x}(t)$ asymptotically converges to $x(t)$.
The fractional-order observer error dynamic equation is given by

$$
D^{\alpha} e(t)=\tilde{A} e(t)+\Phi(x(t), u(t))-\Phi(\hat{x}(t), u(t))-K \xi(t)+F d(t),
$$

where $\tilde{A}=A-L C$ and $e(t)=x(t)-\hat{x}(t)$ is the state observer-based synchronization error.

We consider system (15) and a given set of admissible disturbance signals $\mathcal{D}$. The effects of the external disturbance on the stability error are minimized using the $\mathcal{H}_{\infty}$ norm of $\bar{e}(t)$ and $\xi(t)$ with respect to $d(t)$ as

$$
\|\bar{e}(t)\|+\|\xi(t)\| \leqslant \eta\|d(t)\|,
$$

where $\eta>0$ is a positive number and $\bar{e}(t)$ is the approximation error of $e(t)$.

The $\mathcal{H}_{\infty}$ fractional-order proportional-integral observer design problem presented can be formulated as follows: given the nonlinear fractional-order system (11) and a prescribed level of noise $\eta>0$, find a suitable fractional-order proportionalintegral observer in the form (14), such that :

i) The fractional-order observer error (15) with $d(t)=0$ is asymptotically stable.

ii) Under zero initial condition, the induced $L_{2}$ norm of the operator from $d(t)$ to $\bar{e}(t)$ and $\xi(t)$ is less than $\eta$.

\section{A. FOPI Observer Design}

The following theorem gives sufficient conditions for the fractional-order proportional-integral observer (14) to be stable for $d(t)=0$ and to satisfy the optimality condition (16) for $d(t) \neq 0$.

Theorem 1: If there exist matrices $P=P^{T}>0, W=$ $W^{T}>0, X, V$ and $T>0$ and a positive scalar $\varepsilon_{1}>0$ such that

$$
\left[\begin{array}{cccc}
\Xi & \kappa\left(C^{T} U^{T}-V\right) & \kappa P F & P \\
\cdot & -2 \kappa W+I & 0 & 0 \\
\cdot & \cdot & -\eta^{2} I & 0 \\
\cdot & \cdot & \cdot & -\varepsilon_{1} \kappa^{-1} I
\end{array}\right]<0,
$$

where

$$
\Xi=A^{T} P-C^{T} X+P A-X^{T} C+\varepsilon_{1} \kappa \lambda_{1}^{2} I+I,
$$

and $\lambda_{1}$ is defined in equation (12) and $\kappa>0$ is a given fixed scalar. Then, the error system satisfies the $\mathcal{H}_{\infty}$ performance with given attenuation index $\eta$.

Moreover, the proportional and the integral observer gain matrices are given as

$$
L=P^{-1} X^{T} \text { and } K=P^{-1} V,
$$

with $H=Q^{-1} U$ and $Q=T^{-1} W$.

Proof 1: It follows from lemma 1 that the fractional-order observer error dynamic system (15) can be written as

$$
\left\{\begin{array}{c}
\frac{\partial Z_{1}(\omega, t)}{\partial t}=-\omega Z_{1}(\omega, t)+\tilde{A} e(t)+\Phi(x(t), u(t)) \\
\quad-\Phi(\hat{x}(t), u(t))-K \xi(t)+F d(t)), \\
e(t)=\int_{0}^{\infty} \mu(\omega) Z_{1}(\omega, t) \mathrm{d} \omega,
\end{array}\right.
$$


and its finite-dimensional approximation can be written as follows

$$
\left\{\begin{array}{l}
\frac{\partial \bar{Z}_{1}(\omega, t)}{\partial t}=-\sum_{i=1}^{N} \omega_{i} \psi_{i}(\omega) Z_{1}\left(\omega_{i}, t\right) \\
\quad+\sum_{i=1}^{N} \psi_{i}(\omega)\left(\tilde{A} e(t)+\Pi_{\Phi}-K \xi(t)+F d(t)\right) \\
\bar{e}(t)=\int_{\omega_{\min }}^{\omega_{\max }} \mu(\omega) \bar{Z}_{1}(\omega, t) \mathrm{d} \omega
\end{array}\right.
$$

where $\bar{Z}_{1}(\omega, t)=\sum_{i=1}^{N} \psi_{i}(\omega) Z_{1}\left(\omega_{i}, t\right)$ is the approximation of $Z_{1}\left(\omega_{i}, t\right), \bar{e}(t)$ the approximation of $e(t)$ and $\Pi_{\Phi}=$ $\Phi(x(t), u(t))-\Phi(\hat{x}(t), u(t))$.

The fractional-order filtered output observer-based synchronization error is equivalent to

$$
\left\{\begin{array}{l}
\frac{\partial Z_{2}(\omega, t)}{\partial t}=-\omega Z_{2}(\omega, t)-T \xi(t)+H(y(t)-C \hat{x}(t)), \\
\xi(t)=\int_{0}^{\infty} \mu(\omega) Z_{2}(\omega, t) \mathrm{d} \omega
\end{array}\right.
$$

and its finite-dimensional approximation is given as follows

$$
\left\{\begin{aligned}
& \frac{\partial \bar{Z}_{2}(\omega, t)}{\partial t}=-\sum_{i=1}^{N} \omega_{i} \psi_{i}(\omega) Z_{2}\left(\omega_{i}, t\right) \\
&+\sum_{i=1}^{N} \psi_{i}(\omega)(-T \xi(t)+H(y(t)-C \hat{x}(t)), \\
& \bar{\xi}(t)=\int_{\omega_{\min }}^{\omega_{\max }} \mu(\omega) \bar{Z}_{2}(\omega, t) \mathrm{d} \omega,
\end{aligned}\right.
$$

where $\bar{Z}_{2}(\omega, t)=\sum_{i=1}^{N} \psi_{i}(\omega) Z_{2}\left(\omega_{i}, t\right)$ is the approximation of $Z_{2}\left(\omega_{i}, t\right)$ and $\bar{\xi}(t)$ the approximation of $\xi(t)$.

Let us define the following Lyapunov function as

$$
\begin{aligned}
V(t)=\int_{\omega_{\min }}^{\omega_{\max }} \mu(\omega) \bar{Z}_{1}^{T}(\omega, t) P \bar{Z}_{1}(\omega, t) \mathrm{d} \omega \\
\quad+\int_{\omega_{\min }}^{\omega_{\max }} \mu(\omega) \bar{Z}_{2}^{T}(\omega, t) Q \bar{Z}_{2}(\omega, t) \mathrm{d} \omega .
\end{aligned}
$$

Taking the derivative of equation (23) yields

$$
\begin{aligned}
& \dot{V}=\int_{\omega_{\min }}^{\omega_{\max }} \mu(\omega)\left(-\sum_{i=1}^{N} \omega_{i} \psi_{i}(\omega) Z_{1}^{T}\left(\omega_{i}, t\right)\right. \\
& \left.+\sum_{i=1}^{N} \psi_{i}(\omega)\left(e^{T} \tilde{A}^{T}+\Pi_{\Phi}^{T}-\xi^{T} K^{T}+d^{T} F^{T}\right)\right) P \bar{Z}_{1}(\omega) \mathrm{d} \omega \\
& +\int_{\omega_{\min }}^{\omega_{\max }} \mu(\omega) \bar{Z}_{1}^{T}(\omega) P\left(-\sum_{i=1}^{N} \omega_{i} \psi_{i}(\omega) Z_{1}\left(\omega_{i}, t\right)\right. \\
& \left.+\sum_{i=1}^{N} \psi_{i}(\omega)\left(\tilde{A} e(t)+\Pi_{\Phi}-K \xi+F d\right)\right) \mathrm{d} \omega \\
& +\int_{\omega_{\min }}^{\omega_{\max }} \mu(\omega)\left(-\sum_{i=1}^{N} \omega_{i} \psi_{i}(\omega) Z_{2}^{T}\left(\omega_{i}, t\right)\right.
\end{aligned}
$$

$$
\begin{aligned}
& \left.+\sum_{i=1}^{N} \psi_{i}(\omega)\left(-\xi^{T} T^{T}+e^{T} C^{T} H^{T}\right)\right) Q \bar{Z}_{2}(\omega) \mathrm{d} \omega \\
& +\int_{\omega_{\min }}^{\omega_{\max }} \mu(\omega) \bar{Z}_{2}^{T}(\omega) Q\left(-\sum_{i=1}^{N} \omega_{i} \psi_{i}(\omega) Z_{2}^{T}\left(\omega_{i}, t\right)\right. \\
& \left.+\sum_{i=1}^{N} \psi_{i}(\omega)(-T \xi+H C e)\right) \bar{Z}_{2}(\omega) \mathrm{d} \omega .
\end{aligned}
$$

Using the truncated frequency domain and substitute the term $e(t)$ in (24), one can obtain the following equation

$$
\begin{aligned}
\dot{V} & =-2 \omega_{\min } \int_{\omega_{\min }}^{\omega_{\max }} \mu(\omega) \bar{Z}_{1}(\omega, t) P \bar{Z}_{1}(\omega, t) \mathrm{d} \omega \\
& -2 \omega_{\min } \int_{\omega_{\min }}^{\omega_{\max }} \mu(\omega) \bar{Z}_{2}(\omega, t) Q \bar{Z}_{2}(\omega, t) \mathrm{d} \omega \\
& +\bar{e}^{T}\left[(A-L C)^{T} P+P(A-L C)\right] \bar{e} \\
& +\kappa\left\{\bar{e}^{T} P[\Phi(x, u)-\Phi(\hat{x}, u)]+[\Phi(x, u)-\Phi(\hat{x}, u)]^{T} P \bar{e}\right. \\
& +\xi^{T}\left(Q H C-K^{T} P\right) \bar{e}+\bar{e}^{T}\left(C^{T} H^{T} Q-P K\right) \xi \\
& \left.-2 T \xi^{T} Q \xi+\bar{e}^{T} P F d+d^{T} F^{T} P \bar{e}\right\} .
\end{aligned}
$$

where $\kappa=\max _{\omega \in\left[\omega_{\min }, \omega_{\max }\right]} \sum_{i=1}^{N} \psi_{i}(\omega)>0$.

By using lemma 2 and equation (12), we obtain the following inequality

$$
\begin{aligned}
\bar{e}^{T} P[ & {[(x, u)-\Phi(\hat{x}, u)]+[\Phi(x, u)-\Phi(\hat{x}, u)]^{T} P \bar{e} } \\
& \leqslant \varepsilon_{1}^{-1} \bar{e}^{T} P P \bar{e}+\varepsilon_{1}\|\Phi(x, u)-\Phi(\hat{x}, u)\|^{2} \\
& \leqslant \varepsilon_{1}^{-1} \bar{e}^{T} P P \bar{e}+\varepsilon_{1} \lambda_{1}^{2}\|\bar{e}\|^{2},
\end{aligned}
$$

for any positive number $\varepsilon_{1}$.

Substituting (26) into (25), we obtain the following inequality

$$
\begin{aligned}
& \dot{V} \leqslant-2 \omega_{\min } \int_{\omega_{\min }}^{\omega_{\max }} \mu(\omega) \bar{Z}_{1}(\omega, t) P \bar{Z}_{1}(\omega, t) \mathrm{d} \omega \\
& -2 \omega_{\min } \int_{\omega_{\min }}^{\omega_{\max }} \mu(\omega) \bar{Z}_{2}(\omega, t) Q \bar{Z}_{2}(\omega, t) \mathrm{d} \omega \\
& +\bar{e}^{T}\left[(A-L C)^{T} P+P(A-L C)\right] \bar{e}+\kappa \varepsilon_{1}^{-1} \bar{e}^{T}(t) P P \bar{e}(t) \\
& +\kappa \varepsilon_{1} \lambda_{1}^{2}\|\bar{e}(t)\|^{2}+\kappa \xi^{T}\left(Q H C-K^{T} P\right) \bar{e} \\
& +\kappa \bar{e}^{T}\left(C^{T} H^{T} Q-P K\right) \xi-2 \kappa T \xi^{T} Q \xi+\kappa \bar{e}^{T} P F d \\
& +\kappa d^{T} F^{T} P \bar{e} .
\end{aligned}
$$

Clearly, if

$$
\begin{aligned}
\bar{e}^{T} & {\left[(A-L C)^{T} P+P(A-L C)\right] \bar{e}+\kappa \varepsilon_{1}^{-1} \bar{e}^{T}(t) P P \bar{e}(t) } \\
& +\kappa \varepsilon_{1} \lambda_{1}^{2}\|\bar{e}(t)\|^{2}+\kappa \xi^{T}\left(Q H C-K^{T} P\right) \bar{e} \\
& +\kappa \bar{e}^{T}\left(C^{T} H^{T} Q-P K\right) \xi-2 \kappa T \xi^{T} Q \xi \\
& +\kappa \bar{e}^{T} P F d+\kappa d^{T} F^{T} P \bar{e}<0,
\end{aligned}
$$

then $\dot{V}(t)<0$ which implies that the dynamic system (20) is asymptotically stable i.e the approximation error $\bar{e}(t)$ and the error $e(t)$ converge to zero asymptotically.

Now, we define the $\mathcal{H}_{\infty}$ cost [28] in equation (16) as follows

$$
J=\int_{0}^{\infty}\left(\bar{e}^{T} \bar{e}+\xi^{T} \xi-\eta^{2} d^{T} d\right) \mathrm{d} t
$$


Therefore, we have

$$
J<\int_{0}^{\infty}\left(\bar{e}^{T} \bar{e}+\xi^{T} \xi-\eta^{2} d^{T} d+\dot{V}\right) \mathrm{d} t .
$$

It follows that a sufficient condition for $J \leqslant 0$ is that

$$
\dot{V}+\bar{e}^{T} \bar{e}+\xi^{T} \xi-\eta^{2} d^{T} d \leqslant 0 .
$$

Using inequalities (28) and (31), the sufficient condition can be written as

$$
\left[\begin{array}{l}
\bar{e} \\
\xi \\
d
\end{array}\right]^{T}\left[\begin{array}{ccc}
\Xi_{1} & \kappa\left(C^{T} H^{T} Q-P K\right) & \kappa P F \\
\cdot & -2 \kappa T Q+I & 0 \\
\cdot & \cdot & -\eta^{2} I
\end{array}\right]\left[\begin{array}{c}
\bar{e} \\
\xi \\
d
\end{array}\right]<0
$$

where

$$
\Xi_{1}=(A-L C)^{T} P+P(A-L C)+\kappa \varepsilon_{1}^{-1} P P+\kappa \varepsilon_{1} \lambda_{1}^{2} I+I,
$$

Thus, a sufficient condition for $J \leqslant 0$ is that the following inequality be negative definite

$$
\left[\begin{array}{ccc}
\Xi_{1} & \kappa\left(C^{T} H^{T} Q-P K\right) & \kappa P F \\
\cdot & -2 \kappa T Q+I & 0 \\
\cdot & \cdot & -\eta^{2} I
\end{array}\right]<0 .
$$

Then,

$$
\bar{e}^{T} \bar{e}+\xi^{T} \xi-\eta^{2} d^{T} d \leqslant 0 \rightarrow\|\bar{e}\|+\|\xi\| \leqslant \eta\|d\| .
$$

Using Schur complement, we obtain the following inequality

$$
\left[\begin{array}{cccc}
\Xi_{2} & \kappa\left(C^{T} H^{T} Q-P K\right) & \kappa P F & P \\
\cdot & -2 \kappa T Q+I & 0 & 0 \\
\cdot & \cdot & -\eta^{2} I & 0 \\
\cdot & \cdot & \cdot & -\varepsilon_{1} \kappa^{-1} I
\end{array}\right]<0
$$

where

$$
\Xi_{2}=(A-L C)^{T} P+P(A-L C)+\varepsilon_{1} \kappa \lambda_{1}^{2} I+I .
$$

To convert the above inequality to LMI, let $X=L^{T} P, U=$ $Q H, V=P K$ and $W=T Q$ which implies the following LMI

$$
\left[\begin{array}{cccc}
\Xi & \kappa\left(C^{T} U^{T}-V\right) & \kappa P F & P \\
\cdot & -2 \kappa W+I & 0 & 0 \\
\cdot & \cdot & -\eta^{2} I & 0 \\
\cdot & \cdot & \cdot & -\varepsilon_{1} \kappa^{-1} I
\end{array}\right]<0
$$

where $\Xi=A^{T} P-C^{T} X+P A-X^{T} C+\varepsilon_{1} \kappa \lambda_{1}^{2} I+I$. This ends the proof.

The following theorem gives sufficient conditions for the stability of the fractional-order PI observer (14) when $d(t)=$ 0 .

Theorem 2: There exists an asymptotically convergent fractional-order proportional-integral observer of the form (14) for $d(t)=0$, if there exist matrices $P=P^{T}>0$, $W=W^{T}>0, X, V$ and $T>0$ and a positive scalar $\varepsilon_{1}>0$ such that

$$
\left[\begin{array}{ccc}
\Xi & \kappa\left(C^{T} U^{T}-V\right) & P \\
\cdot & -2 \kappa W+I & 0 \\
\cdot & \cdot & -\varepsilon_{1} \kappa^{-1} I
\end{array}\right]<0
$$

where

$$
\Xi=A^{T} P-C^{T} X+P A-X^{T} C+\kappa \varepsilon_{1} \lambda_{1}^{2} I+I,
$$

$\lambda_{1}$ is defined in equation (12) and $\kappa>0$ is a given fixed scalar.

Moreover, the proportional and the integral observer gain matrices are given as

$$
L=P^{-1} X^{T} \quad \text { and } \quad K=P^{-1} V,
$$

with $H=Q^{-1} U$ and $Q=T^{-1} W$.

Proof 2: We can see that if the LMI (17) of theorem 1 is satisfied then by using the Schur complement we obtain LMI (37) and we have the asymptotic stability for $d(t)=0$. This ends the proof.

\section{B. Fractional-Order Proportional (FOP) Observer Design}

If we are interested in designing the fractional-order proportional observer, then theorem 1 can be changed into the following.

Theorem 3: If there exist matrices $P=P^{T}>0, X$ and a positive scalar $\varepsilon_{1}>0$ such that

$$
\left[\begin{array}{ccc}
\Xi & \kappa P F & P \\
\cdot & -\eta^{2} I & 0 \\
\cdot & \cdot & -\varepsilon_{1} \kappa^{-1} I
\end{array}\right]<0,
$$

where

$$
\Xi=A^{T} P-C^{T} X+P A-X^{T} C+\kappa \varepsilon_{1} \lambda_{1}^{2} I+I,
$$

$\lambda_{1}$ is defined in equation (12) and $\kappa>0$ is a given fixed scalar, then the error system satisfies the $\mathcal{H}_{\infty}$ performance with given attenuation index $\eta$.

Moreover, the proportional observer gain matrix is given as

$$
L=P^{-1} X^{T} .
$$

Proof 3: The proof is similar to the proof of theorem 1, here we omitted it because of page limitation.

\section{Robustness Against Nonlinear UnCERTAinty}

The maximization of the Lipschitz constant maintains the proposed observer robust against some Lipschitz nonlinear uncertainty. In this section, a norm-wise bound on the nonlinear uncertainty is derived. This norm-wise analysis provides an upper bound on the Lipschitz constant of the nonlinear uncertainty and the norm of the Jacobian matrix of the corresponding nonlinear function. The robustness against nonlinear uncertainty presented here is similar to the results proposed in [29]- [32]. Subsequently, we briefly mention the results without proof and refer to those references for detailed discussions and alternative bounds.

Assume a nonlinear uncertainty as follows

$$
\Phi_{\Delta}(x(t))=\Phi(x(t))+\Delta \Phi(x(t)),
$$

where $\Phi_{\Delta}(x(t))$ is uncertain nonlinear function and $\Delta \Phi(x(t))$ is unknown nonlinear uncertainty. Suppose that

$$
\left\|\Delta \Phi\left(x_{1}\right)-\Delta \Phi\left(x_{2}\right)\right\| \leqslant \Delta \lambda_{1}\left\|x_{1}-x_{2}\right\|,
$$

where $\Delta \lambda_{1}$ is the Lipschitz constant.

The following theorem gives sufficient conditions for the stability of the robust fractional-order observer error (15) with 
maximum admissible Lipschitz constant and guarantees also the disturbance attenuation level.

Theorem 4: There exists a robust asymptotically stable adaptive observer of the form (14) with maximum admissible Lipschitz constant $\lambda_{1}^{*}$, if there exist matrices $P=P^{T}>0$, $W=W^{T}>0, X, V$ and $T>0$ and a positive scalar $\varepsilon_{1}>0$ such that

$$
\begin{aligned}
& \max \left(\lambda_{1}\right) \\
& {\left[\begin{array}{cccc}
\Xi & \kappa\left(C^{T} U^{T}-V\right) & \kappa P F & P \\
\cdot & -2 \kappa W+I & 0 & 0 \\
\cdot & \cdot & -\eta^{2} I & 0 \\
\cdot & \cdot & \cdot & -\varepsilon_{1} \kappa^{-1} I
\end{array}\right]<0,}
\end{aligned}
$$

where

$$
\Xi=A^{T} P-C^{T} X+P A-X^{T} C+\varepsilon_{1} \kappa \lambda_{1}^{2} I+I,
$$

and $\lambda_{1}$ is defined in equation (12) and $\kappa>0$ is a given fixed scalar. Then, the error system satisfies the $\mathcal{H}_{\infty}$ performance with given attenuation index $\eta$.

Once the problem is solved, the proportional and the integral observer gains matrices are given as

$$
L=P^{-1} X^{T}, \quad K=P^{-1} V, \quad \text { and } \quad \lambda_{1}^{*}=\max \left(\lambda_{1}\right)
$$

with $H=Q^{-1} U$ and $Q=T^{-1} W$.

Proof 4: The proof is similar to the proof of theorem 1, here we omitted it.

Proposition 1: Suppose that the actual Lipschitz constant of the nonlinear function $\Phi$ system is $\lambda_{1}$ and the maximum admissible Lipschitz constant achieved by theorem 4 is $\lambda_{1}^{*}$. Then, the proposed observer based on theorem 4 can tolerate any additive Lipschitz nonlinear uncertainty with Lipschitz constant $\Delta \lambda_{1}$ such that $\lambda_{1}+\Delta \lambda_{1} \leqslant \lambda_{1}^{*} \longrightarrow \Delta \lambda_{1} \leqslant \lambda_{1}^{*}-\lambda_{1}$.

Proof 5: Based on Schwartz inequality, we have

$$
\begin{aligned}
\left\|\Phi_{\Delta}\left(x_{1}\right)-\Phi_{\Delta}\left(x_{2}\right)\right\| & \left.\leqslant \| \Phi\left(x_{1}\right)-\Phi_{(} x_{2}\right)\|+\| \Delta \Phi\left(x_{1}\right)-\Delta \Phi\left(x_{2}\right) \| \\
& \leqslant \lambda_{1}\left\|x_{1}-x_{2}\right\|+\Delta \lambda_{1}\left\|x_{1}-x_{2}\right\|,
\end{aligned}
$$

According to theorem 4, $\Phi_{\Delta}(x(t))$ can be any Lipschitz nonlinear function with Lipschitz constant less than or equal to $\lambda_{1}^{*}$.

$$
\left\|\Phi_{\Delta}\left(x_{1}\right)-\Phi_{\Delta}\left(x_{2}\right)\right\| \leqslant \lambda_{1}^{*}\left\|x_{1}-x_{2}\right\|
$$

then, there must be

$$
\lambda_{1}+\Delta \lambda_{1} \leqslant \lambda_{1}^{*} \longrightarrow \Delta \lambda_{1} \leqslant \lambda_{1}^{*}-\lambda_{1}
$$

In addition, we have for any continuously differentiable function $\Delta \Phi(x)$

$$
\left\|\Delta \Phi\left(x_{1}\right)-\Delta \Phi\left(x_{2}\right)\right\| \leqslant\left\|\frac{\partial \Delta \Phi}{\partial x}\left(x_{1}-x_{2}\right)\right\|
$$

where $\frac{\partial \Delta \Phi}{\partial x}$ is the Jacobian matrix. Then, $\Delta \Phi(x)$ can be any additive uncertainty with $\left\|\frac{\partial \Delta \Phi}{\partial x}\right\| \leqslant \lambda_{1}^{*}-\lambda_{1}$.

\section{Numerical Simulations}

In this section, numerical results illustrate the performance of the robust fractional-order PI observer design. We compare the performance of the fractional-order PI observer and the fractional-order proportional observer and discuss the simulation results for achieving robust synchronization of non-commensurate fractional-order chaotic Lorenz systems. Grigorenko investigated chaotic dynamics of fractional-order Lorenz system and pointed out that fractional-order Lorenz system displays chaotic behavior for fractional-order $\alpha \geqslant$ 0.9941 in [33]. Its fractional version with external disturbance is described as follows :

$$
\begin{aligned}
& {\left[\begin{array}{l}
D^{\alpha_{1}} x_{1} \\
D^{\alpha_{2}} x_{2} \\
D^{\alpha_{3}} x_{3}
\end{array}\right]=\left[\begin{array}{ccc}
-10 & 10 & 0 \\
28 & -1 & 0 \\
0 & 0 & -\frac{8}{3}
\end{array}\right]\left[\begin{array}{l}
x_{1} \\
x_{2} \\
x_{3}
\end{array}\right]+\left[\begin{array}{c}
0 \\
-x_{1} x_{3} \\
x_{1} x_{2}
\end{array}\right]+\left[\begin{array}{l}
1 \\
1 \\
1
\end{array}\right] d} \\
& {\left[\begin{array}{l}
y_{1} \\
y_{2} \\
y_{3}
\end{array}\right]=\left[\begin{array}{lll}
1 & 0 & 0 \\
0 & 0 & 0 \\
0 & 0 & 1
\end{array}\right]\left[\begin{array}{l}
x_{1} \\
x_{2} \\
x_{3}
\end{array}\right]}
\end{aligned}
$$

where $d(t)=0.05 \sin (60 \pi t)$ is an external disturbance.

The Lorenz system (45) with input disturbance and with integer orders (IO) $\left(\alpha_{1}=\alpha_{2}=\alpha_{3}=1\right)$ and fractional orders (FO) $\left(\alpha_{1}=0.997, \alpha_{2}=0.996\right.$ and $\left.\alpha_{3}=0.998\right)$ has a chaotic attractor as depicted in Fig. 1 where the initial conditions are set to $x_{0}=\left[\begin{array}{lll}1 & 1 & 1\end{array}\right]^{T}$.

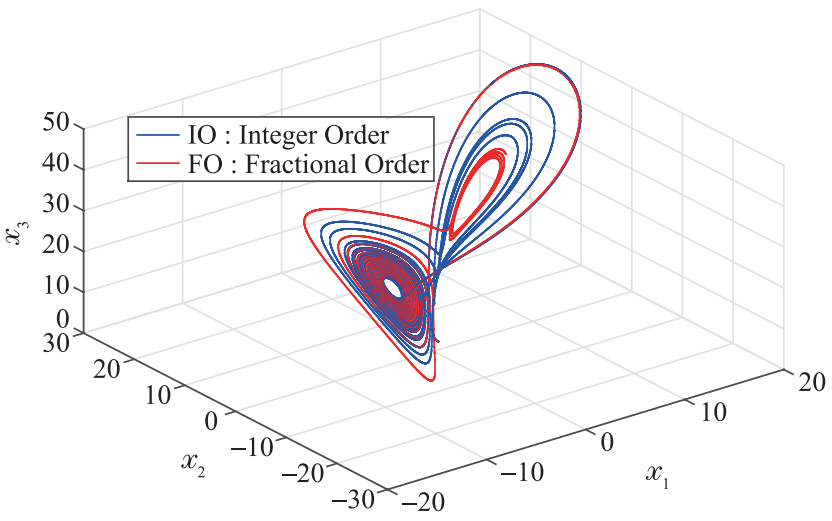

Fig. 1. Chaotic attractors Lorenz system.

The design parameter is chosen such that $\lambda_{1}=0.65$ and $\kappa=1$. From theorem 1 and using the LMI feasibility command "YALMIP" in Matlab, the proportional and the integral observer gains are obtained, respectively,

$L=\left[\begin{array}{ccc}68.10 & 16.26 & 2.34 \\ 129.47 & 16.29 & 8.44 \\ 0.77 & 1.58 & 61.90\end{array}\right], K=\left[\begin{array}{ccc}10.60 & 10.60 & 0 \\ 10.60 & 51.01 & 0 \\ 0 & 0 & 10.60\end{array}\right]$, where $\varepsilon_{1}=0.36$ and $\eta=0.045$.

\section{A. FOPI Observer Versus FPO Observer}

We propose to compare the proposed fractional-order proportional-integral observer (FOPI) to the fractional-order proportional observer (FPO) when modeling errors and external disturbance are present in the system (45).

Figs. 2, 3 and 4 show the trajectories of the states and their estimates, and the synchronization errors are shown in Fig. 5 


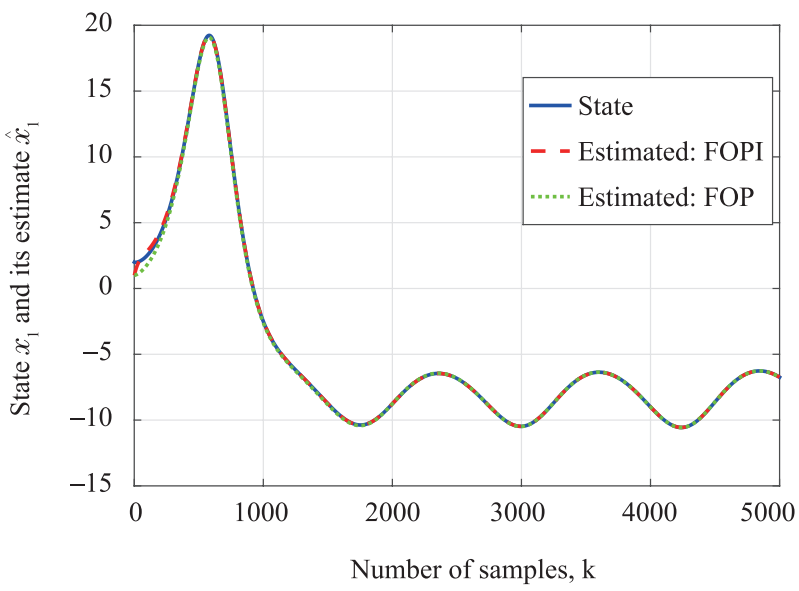

Fig. 2. States $x_{1}^{\mathrm{FOPI}}$ and $x_{1}^{\mathrm{FOP}}$ and their estimates $\hat{x}_{1}^{\mathrm{FOPI}}$ and $\hat{x}_{1}^{\mathrm{FOP}}$ respectively.

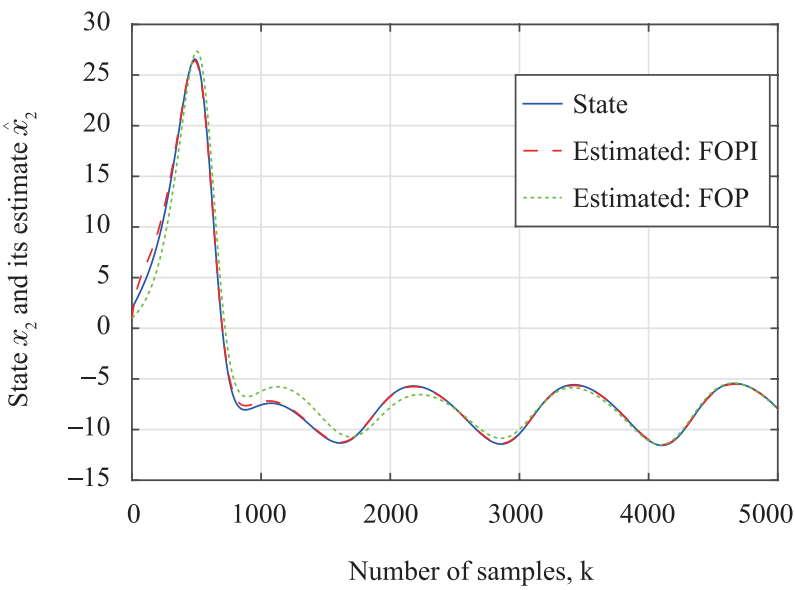

Fig. 3. States $x_{2}^{\mathrm{FOPI}}$ and $x_{2}^{\mathrm{FOP}}$ and their estimates $\hat{x}_{2}^{\mathrm{FOPI}}$ and $\hat{x}_{2}^{\mathrm{FOP}}$ respectively.

for the differentiation orders $\left(\alpha_{1}=0.997, \alpha_{2}=0.996\right.$ and $\left.\alpha_{3}=0.998\right)$. The initial values of FOPI and FPO states are set to $x_{0}=\left[\begin{array}{lll}2 & 2 & 2\end{array}\right]^{T}$ while the initial values of their estimates are $\hat{x}_{0}=\left[\begin{array}{lll}1 & 1 & 1\end{array}\right]^{T}$ where the simulation time is given by $T=2.5 \mathrm{~s}$ and the time step by $h=0.0005$. From the simulations results, the disturbance attenuating properties of the FOPI observer can clearly be observed and the convergence of the estimates is smoother than in the FPO observer. The zero synchronization errors have been achieved which confirm that the proposed observer works well. It should be noted that the proposed FOPI observer is robust against modeling errors and external disturbances. The Root Mean Square (RMS) synchronization errors of FOPI and FOP observers with different differentiation orders have been given in Tables I and II respectively. It can be clearly seen that the synchronization errors achieve better performance with low order fractional systems for both FOPI and FOP observers. Furthermore, the solution for arbitrary initial conditions has been illustrated in Table III. It can be seen that the synchronization errors achieve better performance with low order fractional systems than the normal one. Consequently, these results indicate that smaller the fractional-order is, better are the synchronization errors.

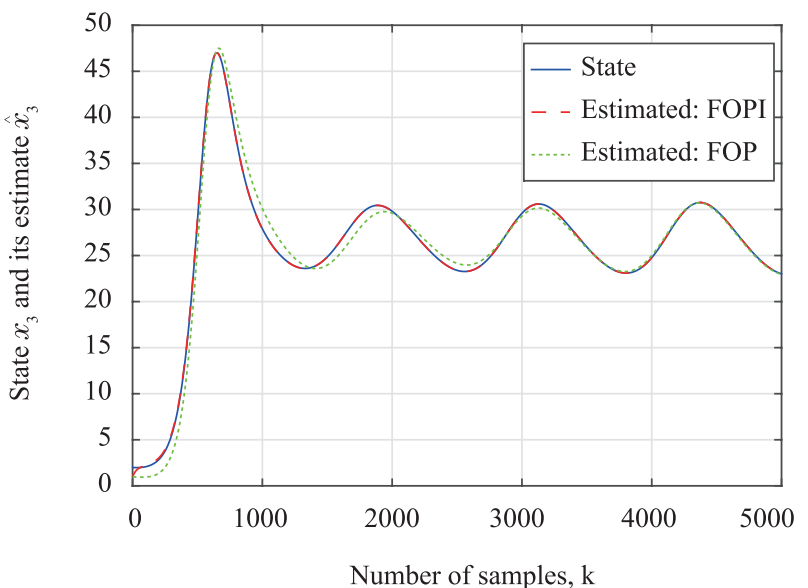

Fig. 4. States $x_{3}^{\mathrm{FOPI}}$ and $x_{3}^{\mathrm{FOP}}$ and their estimates $\hat{x}_{3}^{\mathrm{FOPI}}$ and $\hat{x}_{3}^{\mathrm{FOP}}$ respectively.

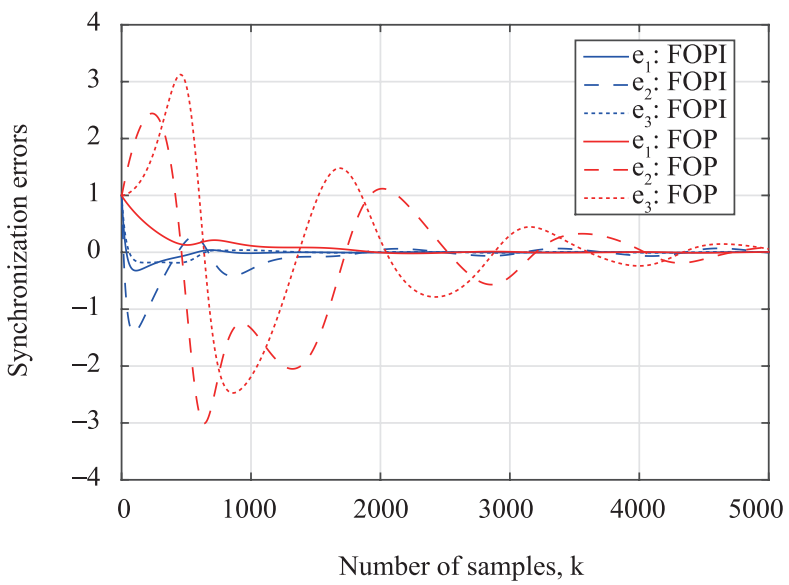

Fig. 5. Synchronization errors.

\section{B. Commensurate Fractional-order (FO) Versus Integer- Order $(I O)$}

We propose to show how the commensurate fractional-order $\alpha$ can affect the performance of the synchronization when external disturbance is present in the system (45). Figs. 6, 7 and 8 show the trajectories of the states and their estimates and the synchronization errors are shown in Fig. 9 for FO $\left(\alpha_{1}=0.995, \alpha_{2}=0.995\right.$ and $\left.\alpha_{3}=0.995\right)$ and IO where the simulation time is given by $T=25 \mathrm{sec}$ and the time step by $h=0.0005$. The initial values of FO and IO states are set to $x_{0}^{\mathrm{FO}}=\left[\begin{array}{lll}2 & 2 & 2\end{array}\right]^{T}$ and $x_{0}^{\mathrm{IO}}=\left[\begin{array}{lll}2 & 2 & 2\end{array}\right]^{T}$ while the initial values of their estimates are $\hat{x}_{0}^{\mathrm{FO}}=\left[\begin{array}{lll}5 & 5 & 5\end{array}\right]^{T}$ and $\hat{x}_{0}^{\mathrm{IO}}=\left[\begin{array}{lll}5 & 5 & 5\end{array}\right]^{T}$ respectively. From the simulations results, the zero synchronization errors have been achieved which confirm that the proposed observer works well. The synchronization errors show that the FOPI can be successfully applied to the chaotic synchronization problem. The RMS synchronization errors of the proposed fractional-order PI observer with commensurate fractional orders have been given in Table IV. It can be seen that the fractional-order $\alpha$ affects also the behavior of the commensurate fractional chaotic dynamical systems and the synchronization starts earlier for smaller values of $\alpha$. 
TABLE I

RMS SYNCHRONIZATION ERRORS OF FOPI OBSERVER WITH DIFFERENT DIFFERENTIATION ORDERS $(T=2.5$ SEC)

\begin{tabular}{lccc}
\hline Differentiation orders & RMS (error 1) & RMS (error 2) & RMS (error 3) \\
\hline (simulated case) $\alpha_{1}=0.997, \alpha_{2}=0.996, \alpha_{3}=0.998$ & 0.0756 & 0.2862 & 0.0744 \\
(decreasing the orders) $\alpha_{1}=0.995, \alpha_{2}=0.996, \alpha_{3}=0.995$ & $\mathbf{0 . 0 7 5 0}$ & $\mathbf{0 . 2 8 4 3}$ & $\mathbf{0 . 0 7 3 6}$ \\
(increasing the orders) $\alpha_{1}=0.999, \alpha_{2}=0.998, \alpha_{3}=0.997$ & 0.0765 & 0.2894 & 0.0746 \\
(integer order case) $\alpha_{1}=1, \alpha_{2}=1, \alpha_{3}=1$ & 0.0772 & 0.2918 & 0.0757 \\
\hline
\end{tabular}

TABLE II

RMS SYNCHRONIZATION ERRORS OF FOP OBSERVER WITH DIFFERENT DIFFERENTIATION ORDERS ( $T=2.5$ SEC)

\begin{tabular}{|c|c|c|c|}
\hline Differentiation orders & RMS (error 1) & RMS (error 2) & RMS (error 3) \\
\hline (simulated case) $\alpha_{1}=0.997, \alpha_{2}=0.996, \alpha_{3}=0.998$ & 0.1736 & 1.1275 & 1.0974 \\
\hline (decreasing the orders) $\alpha_{1}=0.995, \alpha_{2}=0.996, \alpha_{3}=0.995$ & 0.1712 & 1.1019 & 1.0744 \\
\hline (increasing the orders) $\alpha_{1}=0.999, \alpha_{2}=0.998, \alpha_{3}=0.997$ & 0.1760 & 1.1441 & 1.1150 \\
\hline (integer order case) $\alpha_{1}=1, \alpha_{2}=1, \alpha_{3}=1$ & 0.1773 & 1.1728 & 1.1394 \\
\hline
\end{tabular}

TABLE III

RMS SYNCHRONIZATION ERRORS OF FOP I OBSERVER WITH ARBITRARY INITIAL CONDITIONS OF THE DRIVE SYSTEM ( $T=2.5$ SEC)

\begin{tabular}{|c|c|c|c|c|}
\hline Initial conditions & Differentiation orders & RMS (error 1 ) & RMS (error 2) & RMS (error 3) \\
\hline$x_{0}=\left[\begin{array}{lll}2 & 2 & 2\end{array}\right]^{T} \quad($ simulated case $)$ & $\alpha_{1}=1, \alpha_{2}=1, \alpha_{3}=1$ & 0.0772 & 0.2918 & 0.0757 \\
\hline$x_{0}=\left[\begin{array}{lll}5 & 2 & 7\end{array}\right]^{T}$ & $\alpha_{1}=1, \alpha_{2}=1, \alpha_{3}=1$ & 0.2136 & 0.7710 & 0.3400 \\
\hline$x_{0}=\left[\begin{array}{lll}-1 & 1 & 5\end{array}\right]^{T}$ & $\alpha_{1}=0.997, \alpha_{2}=0.996, \alpha_{3}=0.998$ & 0.1090 & 0.2385 & 0.3390 \\
\hline \multirow[t]{2}{*}{$x_{0}=\left[\begin{array}{lll}0.3 & 0.1 & 0.2\end{array}\right]^{T}$} & $\alpha_{1}=0.997, \alpha_{2}=0.996, \alpha_{3}=0.998$ & 0.0781 & 0.2651 & 0.0627 \\
\hline & $\alpha_{1}=1, \alpha_{2}=1, \alpha_{3}=1$ & 0.0799 & 0.2720 & 0.0634 \\
\hline \multirow[t]{2}{*}{$x_{0}=\left[\begin{array}{lll}-0.4 & 0.1 & -0.3\end{array}\right]^{T}$} & $\alpha_{1}=0.997, \alpha_{2}=0.996, \alpha_{3}=0.998$ & 0.0958 & 0.2200 & 0.0832 \\
\hline & $\alpha_{1}=1, \alpha_{2}=1, \alpha_{3}=1$ & 0.0977 & 0.2252 & 0.0841 \\
\hline
\end{tabular}

TABLE IV

RMS SYNCHRONIZATION ERRORS OF FOPI OBSERVER WITH COMMENSURATE ORDERS ( $T=25$ SEC)

\begin{tabular}{|c|c|c|c|}
\hline Commensurate orders & RMS (error 1) & RMS (error 2) & RMS (error 3) \\
\hline$\alpha_{1}=0.995, \alpha_{2}=0.995, \alpha_{3}=0.995$ & 0.2049 & 0.3298 & 0.2037 \\
\hline$\alpha_{1}=0.996, \alpha_{2}=0.996, \alpha_{3}=0.996$ & 0.2061 & 0.3314 & 0.2049 \\
\hline$\alpha_{1}=0.997, \alpha_{2}=0.997, \alpha_{3}=0.997$ & 0.2073 & 0.3330 & 0.2062 \\
\hline (integer order case) $\alpha_{1}=1, \alpha_{2}=1, \alpha_{3}=1$ & 0.2060 & 0.3025 & 0.2056 \\
\hline
\end{tabular}

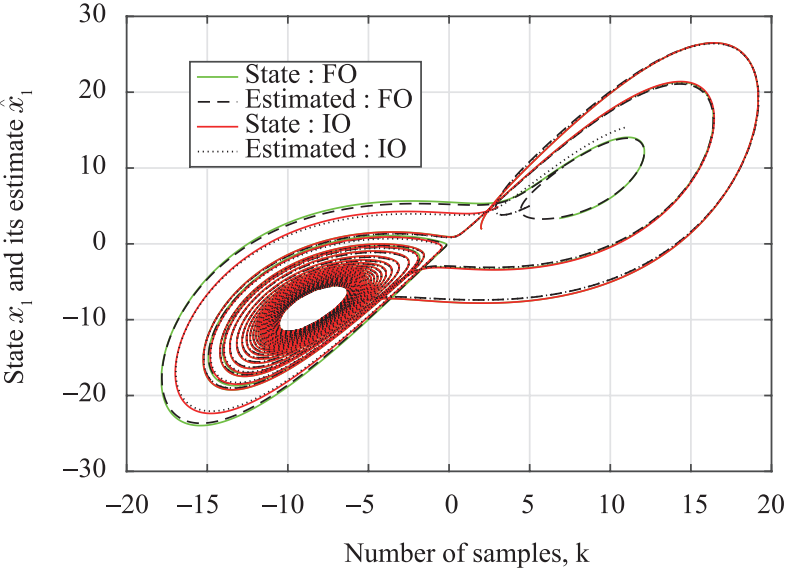

Fig. 6. States $x_{1}^{\mathrm{FO}}$ and $x_{1}^{\mathrm{IO}}$ and their estimates $\hat{x}_{1}^{\mathrm{FO}}$ and $\hat{x}_{1}^{\mathrm{IO}}$ respectively.

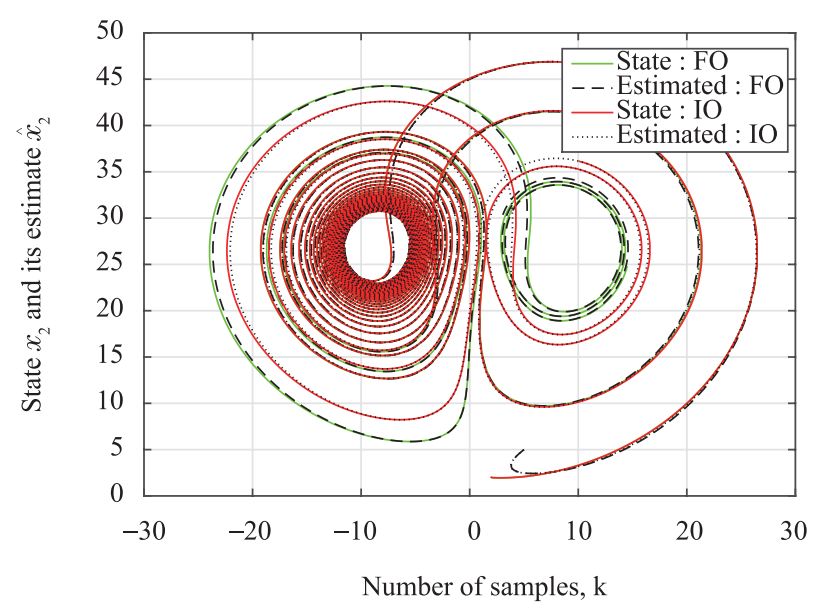

Fig. 7. States $x_{2}^{\mathrm{FO}}$ and $x_{2}^{\mathrm{IO}}$ and their estimates $\hat{x}_{2}^{\mathrm{FO}}$ and $\hat{x}_{2}^{\mathrm{IO}}$ respectively. 


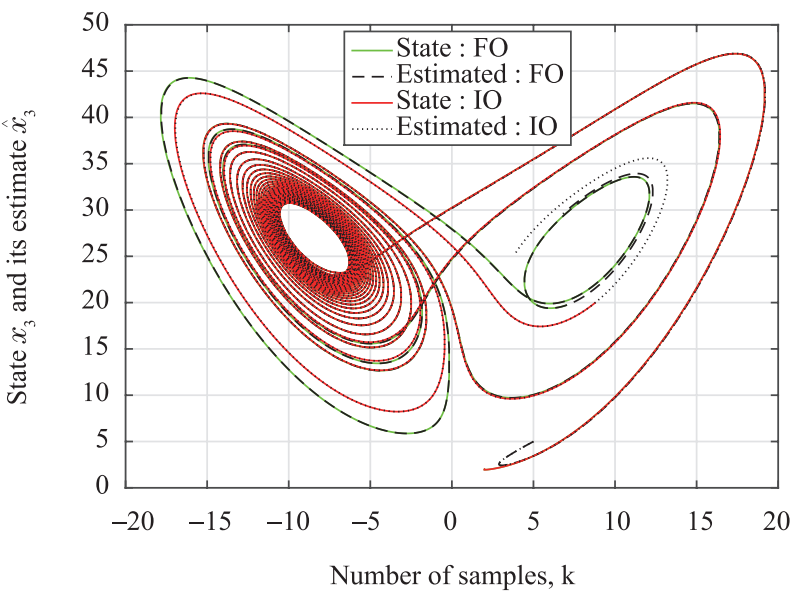

Fig. 8. States $x_{3}^{\mathrm{FO}}$ and $x_{3}^{\mathrm{IO}}$ and their estimates $\hat{x}_{3}^{\mathrm{FO}}$ and $\hat{x}_{3}^{\mathrm{IO}}$ respectively.

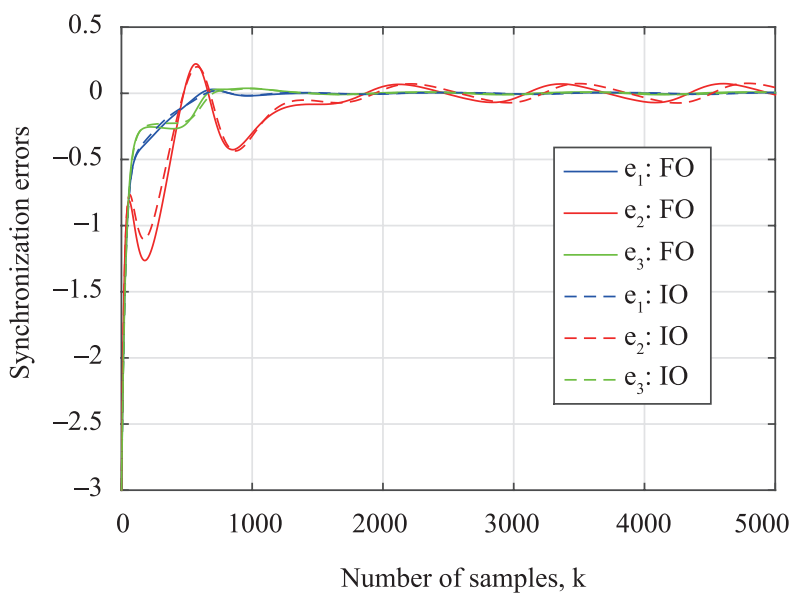

Fig. 9. Synchronization errors.

\section{CONCLUSION}

A robust fractional-order proportional-integral (FOPI) observer-based synchronization has been proposed for nonlinear fractional-order chaotic systems. Sufficient conditions for the convergence of this observer are established in terms of linear matrix inequality (LMI) by using an indirect Lyapunov method. The performance of the FOPI observer allows to achieve good filtering properties in presence of modeling errors and external disturbance. The proposed fractional-order PI observed-based synchronization method can be applicable to a large class of fractional-order complex dynamical networks. It is interesting to find that the fractional-order derivatives and the PI observer gains can be well chosen appropriately to adjust the synchronization effects.

\section{REFERENCES}

[1] R. Martinez-Guerra, C. A. Perez-Pinacho, and G. C. Gómez-Cortes, Synchronization of Integral and Fractional Order Chaotic Systems: A Differential Algebraic and Differential Geometric Approach with Selected Applications in Real-Time. Switzerland: Springer, 2015.

[2] H. Nijmeijer and I. M. Y. Mareels, "An observer looks at synchronization," IEEE Trans. Circ. Syst. I Fund. Theory Appl., vol. 44, no. 10, pp. $882-890$, Oct. 1997.
[3] M. Boutayeb, M. Darouach, and H. Rafaralahy, "Generalized state-space observers for chaotic synchronization and secure communication," IEEE Trans. Circ. Syst. I Fund. Theory Appl., vol. 49, no. 3, pp. 345-349, Mar. 2002.

[4] M. Boutayeb, "Synchronization and input recovery in digital nonlinear systems," IEEE Trans. Circ. Syst. II Express Briefs, vol. 51, no. 8, pp. 393-399, Aug. 2004.

[5] G. P. Jiang, W. X. Zheng, W. K. S. Tang, and G. R. Chen, "Integralobserver-based chaos synchronization," IEEE Trans. Circ. Syst. II Express Briefs, vol. 53, no. 2, pp. 110-114, Feb. 2006.

[6] S. Bhalekar and V. Daftardar-Gejji, "Synchronization of different fractional order chaotic systems using active control," Commun Nonlinear Sci. Numer. Simulat., vol. 15, no. 11, pp. 3536-3546, Nov. 2010.

[7] Y. Tang, Z. D. Wang, and J. A. Fang, "Pinning control of fractionalorder weighted complex networks," Chaos, vol. 19, no. 1, pp. 013112 , Apr. 2009.

[8] X. J. Wu, D. R. Lai, and H. T. Lu, "Generalized synchronization of the fractional-order chaos in weighted complex dynamical networks with nonidentical nodes," Nonlinear Dyn., vol. 69, no. 1-2, pp. 667-683, Jul. 2012.

[9] L. Pan, W. N. Zhou, J. A. Fang, and D. Q. Li, "Synchronization and anti-synchronization of new uncertain fractional-order modified unified chaotic systems via novel active pinning control," Commun Nonlinear Sci. Numer. Simulat., vol. 15, no. 12, pp. 3754-3762, Dec. 2010.

[10] L. Chen, Y. Chai, and R. Wu, "Linear matrix inequality criteria for robust synchronization of uncertain fractional-order chaotic systems," Chaos, vol. 21, no. 4, pp. 043107, Dec. 2011.

[11] O. Morgül and E. Solak, "Observer based synchronization of chaotic systems," Phys. Rev. E, vol. 54, no. 5, 4803-4811, Nov. 1996.

[12] T. L. Liao and N. S. Huang, "An observer-based approach for chaotic synchronization with applications to secure communications," IEEE Trans. Circ. Syst. I Fund. Theory Appl., vol. 46, no. 9, pp. 1144-1150, Sep. 1999.

[13] H. H. Niemann, S. Stoustrup, B. Shafai, and S. Beale, "LTR design of proportional-integral observers," Int. J. Robust Nonlinear Control, vol. 5 , no. 7, pp. 671-693, Nov. 1995.

[14] J. C. Trigeassou, N. Maamri, J. Sabatier, and A. Oustaloup, "A Lyapunov approach to the stability of fractional differential equations," Signal Process., vol. 91, no. 3, pp. 437-445, Mar. 2011.

[15] M. R. Rapaić and A. Pisano, "Variable-order fractional operators for adaptive order and parameter estimation," IEEE Trans. Autom. Control, vol. 59, no. 3, pp. 798-803, Mar. 2014.

[16] E. A. Boroujeni and H. R. Momeni, "Non-fragile nonlinear fractional order observer design for a class of nonlinear fractional order systems," Signal Process., vol. 92, no. 10, pp. 2365-2370, Oct. 2012.

[17] Y. H. Lan and Y. Zhou, "Non-fragile observer-based robust control for a class of fractional-order nonlinear systems," Syst. Control Lett., vol. 62, no. 12, pp. 1143-1150, Dec. 2013.

[18] I. ND́oye, H. Voos, and M. Darouach, "Observer-based approach for fractional-order chaotic synchronization and secure communication," IEEE J. Emerg. Sel. Top. Circ. Syst., vol. 3, no. 3, pp. 442-450, Sep. 2013. 
[19] I. Podlubny, Fractional Differential Equations. New York, USA: Academic Press, 1999.

[20] S. Das, Functional Fractional Calculus for System Identification and Controls. Berlin Heidelberg, Germany: Springer, 2008.

[21] G. Montseny, "Diffusive representation of pseudo-differential timeoperators, in ESAIM : Fractional Differential Systems: Models, Methods and Applications, vol. 5, pp. 1318-1326, 1998.

[22] J. Sabatier, C. Farges, and J. C. Trigeassou, "Fractional systems state space description: Some wrong ideas and proposed solutions," J. Vibrat. Control, vol. 20, no. 7, pp. 1076-1084, Jul. 2014.

[23] D. Heleschewitz and D. Matignon, "Diffusive realizations of fractional integro-differential operators: structure analysis under approximation, in IFAC Conf. System, Structure and Control, vol. 2, pp. 243-248, 1998.

[24] J. C. Trigeassou and N. Maamri, "Initial conditions and initialization of linear fractional differential equations," Signal Process., vol. 91, no. 3, pp. 427-436, Mar. 2011

[25] D. Matignon and B. dÁndra-Novel, "Observer-based controllers for fractional differential systems, in Proc. 36th Conf. Decision and Control, San Diego, USA, vol. 5, pp. 4967-4972, 1997.

[26] M. Darouach, M. Zasadzinski, and S. J. Xu, "Full-order observers for linear systems with unknown inputs," IEEE Trans. Autom. Control, vol. 39, no. 3, pp. 606-609, Mar. 1994.

[27] P. P. Khargonekar, I. R. Petersen, and K. Zhou, "Robust stabilization of uncertain linear systems: Quadratic stabilizability and $H_{\infty}$ control theory," IEEE Trans. Autom. Control, vol. 35, no. 3, pp. 356-361, Mar. 1990.

[28] S. Boyd, L. El Ghaoui, E. Féron, and V. Balakrishnan, Linear Matrix Inequalities in Systems and Control Theory. Philadelphia, USA: SIAM, 1994.

[29] M. Abbaszadeh and H. J. Marquez, "Robust $H_{\infty}$ observer design for a class of nonlinear uncertain systems via convex optimization," in Proc. IEEE American Control Conf., New York, USA, 2007, pp. 1699-1704.

[30] M. Abbaszadeh and H. J. Marquez, "LMI optimization approach to robust $H_{\infty}$ observer design and static output feedback stabilization for discrete-time nonlinear uncertain systems," Int. J. Robust Nonlinear Control, vol. 19, no. 3, pp. 313-340, Feb. 2009.

[31] M. Abbaszadeh and H. J. Marquez, "Dynamical robust $H_{\infty}$ filtering for nonlinear uncertain systems: An LMI approach," J. Franklin Inst., vol. 347 , no. 7 , pp. $1227-1241$, Sep. 2010.

[32] M. Abbaszadeh and H. J. Marquez, "A generalized framework for robust nonlinear $H_{\infty}$ filtering of Lipschitz descriptor systems with parametric and nonlinear uncertainties," Automatica, vol. 48, no. 5, pp. 894-900, May 2012
[33] I. Grigorenko and E. Grigorenko, "Chaotic dynamics of the fractional Lorenz system,” Phys. Rev. Lett., vol. 91, no. 3, pp. 034101, Jul. 2003.

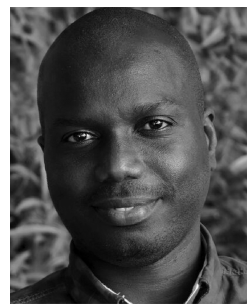

Ibrahima N'Doye is a Postdoc at King Abdullah University of Science and Technology (KAUST) in the Division of Computer, Electrical and Mathematical Sciences and Engineering (CEMSE). He received simultaneously the Ph.D. degree in Automatic Control from University of Lorraine at the Research Center of Automatic Control (CRAN-CNRS, University of Lorraine), France and University Hassan II Ain Chock, Casablanca, Morocco, in 2011. From 2012 to 2014, he was a Postdoc at University of Luxembourg in the Faculty of Science, Technology and Communication, Research Unit of Engineering Sciences. His research interests are in the area of estimation and control of fractional-order systems and nonlinear dynamical systems with applications in engineering and biomedical fields.

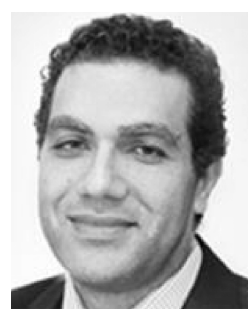

Khaled Nabil Salama (S'97 - M'05 - SM'10) received the B.S. degree (with Hons.) from the Department of Electronics and Communications, Cairo University, Giza, Egypt, in 1997, and the M.S. and Ph.D. degrees from the Department of Electrical Engineering, Stanford University, Stanford, CA, USA, in 2000 and 2005, respectively. He was an Assistan Professor with the Rensselaer Polytechnic Institute, Troy, NY, USA, from 2005 to 2009. In 2009, he joined the King Abdullah University of Science and Technology, Thuwal, Saudi Arabia, in 2009, where he was the founding Program Chair until 2011.

He is currently an Associate Professor with the King Abdullah University of Science and Technology. His focus on CMOS sensors for molecular detection has been funded by the National Institutes of Health and the Defense Advanced Research Projects Agency. He has authored over 200 papers and holds 20 patents on low-power mixed-signal circuits for intelligent fully integrated sensors and nonlinear electronics specially memristor devices.

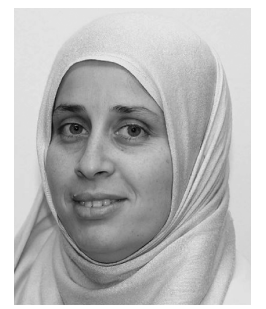

Taous Meriem Laleg-Kirati is an assistant professor in the division of Computer, Electrical and Mathematical Sciences and Engineering at KAUST. She joined KAUST in December 2010. From 2009 to 2010 , she was working as a permanent research scientist at the French Institute for research in Computer Sciences and Control Systems (INRIA) in Bordeaux. She earned her Ph.D. in Applied Mathematics from INRIA Paris, in 2008. She holds a Master in control systems and signal processing from University Paris 11 in France. Her research interests include, modeling, estimation, and control of infinite dimensional (distributed) systems including partial differential equations systems and fractional systems, and signal/image analysis. She considers applications in engineering and biomedical fields. Webpage: http://emang.kaust.edu.sa. 